\title{
HUMAN RESPONSE TO SINGLE AND COMBINED SINUSOIDAL VERTICAL VIBRATION
}

\author{
Lauren Gant*, David Wilder, Donald Wasserman \\ Jolt/Vibration/Seating Lab, Iowa Spine Research Center, \\ 1408 SC, The University of Iowa, Iowa City, Iowa 52242, USA \\ lau ren-graupner@uiowa.edu, david-wilder@uiowa.edu, \\ dewasserman@juno.com
}

\section{Introduction}

Task performance decrement has been reported with exposure to combined vibration by Cohen et al, 1977. ${ }^{1}$ In that study subjects were exposed to 3 different sinusoidal vertical vibration conditions: $2.5 \mathrm{~Hz}, 5 \mathrm{~Hz}$, and a combination of $2.5 \mathrm{and} 5 \mathrm{~Hz}$, and asked to complete a task using all four limbs. It was shown that performance was the worst in the combination of 2.5 and $5 \mathrm{~Hz}$. Although the study provides insight into human performance response to complex vibration, it does not provide any physiological or biomechanical measurements. The current study uses Cohen et al.'s methodology as a foundation, but includes collection of electromyography (EMG) to capture muscle activity. This study may explain the substantial decrease in performance during exposure to the combined vibration.

\section{Methods}

Fourteen, right-handed males volunteered for the study. The participants were exposed to three trials of four vertical vibration conditions: non-vibration control, vibration at $2.5 \mathrm{~Hz}$, vibration at $5 \mathrm{~Hz}$, and vibration combining $2.5 \mathrm{~Hz}$ and $5 \mathrm{~Hz}$. Each vibration condition had an acceleration of $0.69 \mathrm{~ms}^{-2} \mathrm{rms}$, and lasted for 30 seconds per exposure. The vibrations were produced with a six-degree-of-freedom Hydraudyne motion platform (Bosch-Rexroth, Netherlands). During exposure, the participants sat upright in a steel tractor seat with no back support or physical postural reminders. The seat and posture assumed by the participants was identical to those in the Cohen et al. study. The subjects were asked to complete a simple four-limb task during the testing in order to resemble the previous study. ${ }^{1}$ Surface electromyography (EMG) was used to capture the muscle activity of the left and right lumbar erector spinae (ES) muscles because the erector spinae muscles are primarily responsible for support during forward flexed tasks. ${ }^{2}$ Two Ag-AgCl bipolar electrodes (Model D-100, Therapeutics Unlimited, Iowa City, IA, USA) with built in pre-amplification were placed on the left and right lumbar ES. Back muscle (EMG) activity was calibrated, recorded and ensembleaveraged. In order to compare information between subjects, a normalization taking body weight into account was performed. Two-way analysis of variance (ANOVA) was performed for main effects and interactions. The factors were "vibration condition," with four levels, and "participant," with 14 levels. The mean EMG voltages, the peak-to-peak EMG voltages, and the reaction times were examined. 


\section{Results}

The mean rectified and smoothed EMG activity differed significantly between participants for the right $\mathrm{ES}(\mathrm{p}=0.000)$, but not for the left $\mathrm{ES}(\mathrm{p}=0.524)$. In terms of peak-to-peak rectified and smoothed EMG activity in the left ES, the interaction between participants and environment was significant $(\mathrm{p}=0.000)$. Differences detected via paired $\mathrm{t}$-test between peak-to-peak left and right ES activity was significant $(\mathrm{p}=0.041)$. Analysis of the right ES activity showed that the response delay differed significantly with environment $(\mathrm{p}=0.03)$.

\begin{tabular}{|c|c|c|c|c|c|c|}
\hline \multicolumn{7}{|c|}{ Vertical Vibration Condition } \\
\hline \multicolumn{2}{|c|}{$2.5 \mathrm{~Hz}$} & \multicolumn{2}{c|}{$5 \mathrm{~Hz}$} & \multicolumn{2}{c|}{$2.5 \mathrm{~Hz}$ and $5 \mathrm{~Hz}$} \\
\hline Response & \multicolumn{2}{|c|}{} & \multicolumn{2}{c|}{} \\
\hline Erector Spinae & Left & Right & Left & Right & Left & Right \\
\hline Frequency & $2.5 \mathrm{~Hz}$ & $2.5 \mathrm{~Hz}$ & $5 \mathrm{~Hz}$ & $5 \mathrm{~Hz}$ & $2.5 \mathrm{~Hz}$ & $2.5 \mathrm{~Hz}$ \\
\hline No. Participants & 6 & 2 & 8 & 3 & 5 & 3 \\
\hline
\end{tabular}

Table 1. Frequency responses and number of participants (out of 14) who responded cyclically to each vibration condition

Not all subjects responded to the vibration frequency (Table 1). The left (L) erector spinae responded more often than the right $(\mathrm{R})$ erector spinae. Both sides only responded at $2.5 \mathrm{~Hz}$ to the combined vibration. Chronic involuntary exercise of the muscles opposite the dominant hand could explain why the left erector spinae responded more often than the right. ${ }^{3}$ A balanced posture with respect to the vertical acceleration could explain the lack of response in many subjects. Responding only at $2.5 \mathrm{~Hz}$ to a combined signal would allow an acceleration component at $5.0 \mathrm{~Hz}$, the seated human's natural frequency, to apply forces to the spine. This could explain both the performance decrement noted above. Cohen, Wasserman and Hornung's 1977 study, the basis for the current study, demonstrated that exposure to the combination of $2.5 \mathrm{~Hz}$ and $5 \mathrm{~Hz}$ resulted in decreased performance. The lack of response to the $5 \mathrm{~Hz}$ component of the input signal is likely correlated with the performance degradation they found.

\section{Discussion}

Handedness has a significant effect on erector spinae response and poses the possibility of asymmetric mechanical trunk control. This study has also revealed a significant musculoskeletal control system limitation, raising que stions about the ability of the human to cope with complex vibration environments.

\section{References}

1. Cohen H, Wasserman D, Hornung R (1977). Human performance and transmissibility under sinusoidal and mixed vertical vibration. Ergonomics. 20(3): 207-216.

2. Seroussi R, Pope M (1987). The relationship between trunk muscle electromyography and lifting moments in the sagittal and frontal planes. Journal of Biomechanics. 20: 135-146.

3. Sung P, Spratt K, Wilder D (2004) A possible methodological flaw in comparing dominant and nondominant sided lumbar spine mucle responses without simultaneously considering hand dominance. Spine. 29: 1914-1922. 